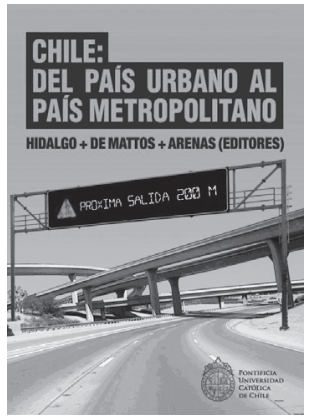

\title{
Rodrigo Hidalgo, Carlos de Mattos y Federico Arenas (editores). Chile: del país urbano al país metropolitano
}

\author{
Santiago: Pontificia Universidad Católica de Chile, \\ Instituto de Geografía, Instituto de Estudios Urbanos y \\ Territoriales, Serie GEOlibros 12, 2009, 469 p.
}

\author{
Maribel Carreño ${ }^{1}$
}

El proceso de desarrollo que viven las ciudades, producto de la constante intervención del ser humano en pro de su bienestar y de la satisfacción de sus necesidades, ha generado un imparable proceso de transformación, cuyas consecuencias se han evidenciado en diversas ciudades chilenas. El fenómeno que da inicio a este proceso de transformación está ligado a los notables cambios económicos acaecidos en el país que, a su vez, están relacionados con el proceso de globalización. Más allá de las causas, que se muestran más homogéneas, el estudio se centra también en las consecuencias y en los cambios observables en las ciudades. "Chile: del país urbano al país metropolitano" es un obra que se encarga de aclarar los efectos que ha provocado la evolución de un proceso de urbanización a uno de metropolización, variaciones como la especialización productiva del territorio, su segregación, diversificación y hasta los cambios generados en el medio ambiente. El texto se encuentra integrado por 24 capítulos, agrupados en 5 secciones que son capaces de entregar de modo ordenado y estructurado la información requerida para comprender la metropolización en ciudades como Antofagasta, Valparaíso y Concepción.

El texto comienza con la sección "Transformaciones sociodemográficas, económicas y ambientales recientes del sistema urbano nacional", la que se inicia con el trabajo de

1 Estudiante de geografía que cursa su segundo año de carrera. Instituto de Geografía, Pontificia Universidad Católica de Chile (Chile). E-mail: mdcarren@uc.cl
Severino Escolano y Jorge Ortiz, quienes analizan una tipología de diversas funciones económicas presentes en las ciudades, que combinadas y emplazadas en un territorio particular, generan patrones de actividad económica urbana. El estudio se extiende a partir del tamaño demográfico de las ciudades, el que permitirá que se origine un campo de diversidad sistemática para dichas tendencias.

Posteriormente son Marcelo Lufin Varas y Miguel Atienza Úbeda quienes proponen un análisis ocupacional de las capitales regionales de Chile, con el propósito de profundizar, comprender y caracterizar la estructura de ocupaciones características de esas zonas. El análisis está especialmente dirigido a la evolución intercensal de las concentraciones ocupacionales que suelen presentarse de manera concentrada en el espacio. Estas son las que están principalmente relacionadas con el conocimiento, ciencia, arte, gerencia y cultura, que tienden a ser menos analizadas, en pro de estudios de distribución de actividades productivas, que tienden a distribuirse más homogéneamente en el país.

Jorge Rodríguez, Daniela González y Miguel Ojeda dan paso al siguiente estudio centrado en el fenómeno de urbanización en las ciudades chilenas. Basados en el sistema de ciudades como causa de la mutación de las formas de asentamiento de la población, afirman que estas formas son diversas y que los sistemas se mueven de macrocefálicos a multipolares. Sin embargo, el verdadero foco de estudio de este trabajo es la diversidad presente entre estos polos, en 
otras palabras, la variabilidad sociodemográfica interna del sistema urbano en Chile. Los autores narran la evolución del sistema de ciudades nacional a partir del año 1950, afirmando que dependiendo del tamaño de las ciudades, se presentará un bienestar social, una dinámica y una distribución poblacional urbana característica.

Para dar fin al primer capítulo sobre las transformaciones en los sistemas de las ciudades chilenas, Hugo Romero, Claudio Moscoso y Pamela Smith estudian una de las consecuencias de este proceso, centrándose en la expansión urbana, el cambio estructural y la diversidad funcional sufrida por las ciudades chilenas, factores que se traducen en la sustentabilidad ambiental. Al ser asumidos los riesgos y consecuencias de la metropolización, se hace patente la necesidad de tomar medidas necesarias frente a ellos. El estudio enfatiza la insustentabilidad ambiental de los procesos de urbanización y las precarias condiciones ambientales de las poblaciones vulnerables y de escasos recursos del país. Todo este fenómeno genera segregación socioespacial, traducida en una segregación socioambiental.

La segunda unidad de la obra se titula "Las ciudades del norte: metropolización y nueva geografía social". Esta sección comienza con el estudio de Iván Fernández Vergara y Miguel Atienza Úbeda, los que a través de un estudio histórico-económico de la ciudad de Antofagasta, la establecen como el foco de concentración más importante del norte de Chile. A partir del estudio histórico explican la aglomeración industrial y urbana en la zona, el cual también contribuye a dejar de lado las limitaciones que origina la nueva geografía económica. Los autores realizan también un análisis sobre la sustentabilidad económica de los centros mineros ubicados en la ciudad de estudio.

Continuando la temática de la metropolización en la ciudad de Antofagasta, son Óscar Figueroa y Claudia Contreras quienes investigan los efectos espaciales que ha ocasionado este proceso en el desarrollo urbano y el crecimiento de la ciudad. Antofagasta se muestra como un epicentro de atracción poblacional, producto de un importante desarrollo económico generado por los excedentes de la minería, que se han demostrado en el desarrollo urbano y el crecimiento comercial, la construcción de nuevos barrios residenciales, edificaciones en altura en la periferia y la presencia de mayores salarios. A partir de estas características la ciudad de Antofagasta comienza a jugar un rol de importancia frente al resto de las urbes del país, y con su paulatino crecimiento es capaz de situarse en un lugar relevante en la competencia nacional.

En el siguiente apartado, Óscar Figueroa y Luis Fuentes realizan un estudio centrado en las ciudades de Iquique-Alto Hospicio y su desarrollo como foco de atracción nacional e internacional. Se asume que Iquique, una urbe ya consolidada, tiene en su poder la aglomeración de actividades económicas, extractivas y turísticas, mientras que en la comuna de Alto Hospicio se ha advertido un notorio crecimiento. Si bien ambas presentan diferencias en la conformación de su población en términos socioeconómicos, pues una concentra los estratos sociales medios y altos y la otra solo los bajos, en conjunto conforman un sistema urbano-social completo. Ahora bien, es producto de estos desarrollos que se ha hecho evidente la aparición de problemas en torno a la expansión urbana de estos centros: desarrollo inmobiliario en la periferia, fragmentación social y operativa en la zona. Se hace patente la necesidad de prestar atención a las gestiones urbanas con el fin de evitar estas problemáticas.

Para concluir este apartado, Rodrigo Hidalgo, Federico Arenas y Rafaella Monsalve desarrollan un estudio que trata sobre las causas de la conurbación Coquimbo-La Serena, evidenciando fenómenos como el cambio en el funcionamiento externo e interno de la ciudad, y lo que es más notorio, la estructura urbana: edificación de barrios cerrados y presencia evolutiva de malls. En definitiva, los cambios acaecidos en la ciudad no solo buscan satisfacer problemas internos, además se pretende generar la posibilidad de un mejoramiento en las posibilidades de desarrollo urbano.

La tercera sección del libro titulada "Vectores de cambio del Área Metropolitana de Santiago, Valparaíso y Concepción", se inicia 
con el texto de Melandra Molina, Hugo Romero y Pablo Sarricolea, que tiene como temática central la expansión metropolitana de Santiago y Valparaíso y las distintas maneras en que el proceso se expresa, lo que depende de las diferencias geográficas del lugar de emplazamiento. En los diferentes patrones generados por el proceso se presenta una característica constante de relevancia: el uso indiscriminado del suelo y los recursos del mismo. Esta medida trae como consecuencia efectos sobre el nivel de bienestar de la población (cambios en las temperaturas y contaminación). Se concluye una relación indirecta entre la calidad ambiental y el desarrollo económico de la ciudad.

El siguiente estudio es dirigido por Eliana Muga y Marcela Rivas, quienes desarrollan una investigación sobre el Área Metropolitana de Valparaíso (AMV) y la transformación de su estructura y paisaje urbano. En este trabajo se investiga el impacto que origina la nueva dinámica urbana. También se le otorga relevancia a las particularidades observables en el AMV, su rol como ciudadpuerto, su estructura y la conducta histórica de sus habitantes. A través de esta investigación no solo se accede a conocer los factores que impulsan la metropolización y los efectos que origina, además se da el impulso para generar nuevas instancias que ayuden a amortiguar los cambios de las estructuras urbanas.

Posteriormente, continúa el trabajo de Hugo Marcelo Zunino y Rodrigo Hidalgo, enfatizando las consecuencias que generan los cambios en las estructuras. Además se presta atención a la distribución de los conjuntos de vivienda social, que son producto de las políticas empleadas durante el período 19902005, en la ciudad de Valparaíso. En la investigación se hace notoria la incorporación de políticas económicas neoliberales, adoptadas en el período del gobierno militar y que se mantienen vigentes durante los gobiernos posteriores. Los principales efectos observables por los autores son: el desarrollo urbano-regional y la segregación residencial. Esta última se demuestra en la proliferación de barrios cerrados y ciudades valladas en la periferia de la urbe, la que otorga un distanciamiento entre las diferentes clases sociales, ya sea voluntaria o forzada.
A continuación, se realiza un estudio sobre los agentes que intervienen en la manifestación de los diferentes patrones de metropolización de las ciudades. Este trabajo es realizado por Sergio Baeriswyl Rada, quien establece que las tendencias de metropolización suelen ser efecto combinado de las políticas, medidas económicas y variables sociales. El centro de estudio es el Área Metropolitana de Concepción (AMC), la que es calificada como un caso especial, pues tiene la característica de poseer un crecimiento desigual, ya que depende del espacio jurisdiccional comunal que tiende a tomar medidas espaciales diferentes. Finalmente se afirma que los patrones de metropolización y el desarrollo urbano son consecuencia de factores múltiples y un tanto indeterminados y variables, pero que terminan por definir su crecimiento.

Siguiendo en el mismo apartado, Carolina Rojas Quezada, Sergio Opazo Saldivia y Edilia Jaque Castro continúan con el estudio de la ciudad de Concepción. La investigación se encarga de descubrir las diferentes modalidades de crecimiento del AMC, de modo de establecer su estructura dispersa o compacta. A través de variados métodos, son capaces de establecer si el AMC sigue un proceso ligado a la complejidad, a la dispersión o a la fragmentación del territorio.

Para dar fin a la tercera sección en curso, Leonel Pérez Bustamante y Edison Salinas Varela presentan un trabajo en que se manifiesta la importancia del AMC, como segundo centro importante después del Área Metropolitana de Santiago. Es por eso que el estudio se concentra en los nuevos patrones de crecimiento de la ciudad de Concepción durante el período 1992-2002, mostrando las nuevas estructuras generadas y las nuevas formas de crecimiento sufridas en la ciudad producto de los procesos de cambio a nivel nacional.

En el siguiente apartado del libro, "Las ciudades del centro-sur: reestructuración productiva, morfología urbana y huella ecológica", se presenta un estudio dirigido por Federico Arenas, Rodrigo Hidalgo y Gastón Aliaga, basado en ciertas ciudades medias de la zona central de Chile. Estas urbes, a pesar de tener un patrón de expansión simi- 
lar, crecen de manera más notoria que otros centros urbanos medios de mayor tamaño. En el texto también se presenta un estudio en el que, a través de un Índice de DesarroIlo Socioeconómico, se lleva a cabo una investigación sobre las nuevas conurbaciones que se han desarrollado en las ciudades medias de pequeño tamaño.

Como parte del mismo capítulo, Carolina Negrete Rodríguez y Rodrigo Hidalgo realizan un trabajo donde se presenta una de las nuevas formas de urbanización conocido como los barrios cerrados. El impacto generado por este fenómeno se representa por la consecuencia más relevante de este proceso: la segregación socioespacial. El estudio se realizó a partir de cinco comunas del valle del Aconcagua, Región de Valparaíso, donde se recopiló información sobre el nivel socioeconómico de los habitantes de los barrios cerrados y se analizó la distribución de los distintos estratos sociales en la ciudad y los verdaderos impactos que estas modalidades inmobiliarias generan en la población.

Luego, Federico Arenas, Daniela González Espinoza y Gastón Aliaga continúan con el estudio de ciudades medias de Chile, con el propósito de caracterizar el crecimiento urbano. Se toma como área de estudio la conurbación Rancagua-Machalí en contraste con la ciudad de Santiago, buscando similitudes y diferencias en sus procesos de desarrollo. La necesidad de este trabajo encuentra su justificación en el comportamiento diferenciado que tienen estas urbes respecto del resto de las ciudades medias presentes en Latinoamérica, pues estas últimas poseen una velocidad de crecimiento demográfico mayor. A pesar de ello, la superficie de expansión es mayor en las ciudades medias chilenas. De este modo se pretende establecer causas que caractericen el fenómeno de desarrollo urbano en este tipo de centros urbanos.

Por su parte, Andrés Maragaño presenta en su trabajo un estudio sobre la ciudad de Talca y sus transformaciones ligadas a los cambios en las políticas de uso del suelo, que se han liberalizado. El autor estudia los cambios de esta ciudad en distintos niveles, desde su organización espacial hasta la reconfiguración cívica; todo bajo la consigna de que las ciudades de Chile se definen a partir de sus expansiones y provocan la adquisición de nuevos roles y estructuras urbanas.

Para dar fin a esta sección, Cristian Henríquez Ruiz investiga el proceso de perifragmentación del espacio urbano de la conurbación Chillán-Chillán Viejo, lo que corresponde a un estudio de la fragmentación del paisaje y permite observar las consecuencias, tanto físicas como sociales, del proceso de urbanización. En la conurbación Chillán-Chillán Viejo se ha identificado la aparición paulatina de condominios privados, parcelas de agrado, villas e industrias, entre otros, que han originado un crecimiento fragmentado de la zona. A su vez, esta división de la ciudad se presenta como característica de aquellos centros urbanos poco sustentables, ya que la relación entre ambos factores -fragmentación y sustentabilidad- es de carácter indirecto, pues las ciudades compactas son determinadas como las confortables para la población.

"Las ciudades del sur: expansión urbana, reestructuración productiva y huella ecológica" corresponde a la quinta y última unidad del texto. La sección comienza con el trabajo de Fernando Peña y Miguel Escalona, quienes se interesan por la intercomuna de La Araucanía centro y su proceso de expansión urbana. La problemática abordada se desarrolla en torno a la proliferación de nuevos proyectos inmobiliarios que se emplazan en los límites de las comunidades indígenas ubicadas en la zona, generando la necesidad de una planificación acorde que asegure el bienestar de los habitantes, evitando conflictos sociales y desgastes ambientales.

A continuación, Laura Rodríguez, Elisa Cordero, Gustavo Rodríguez y Cristián Gutiérrez presentan en su texto la visión de la ciudad como palimpsesto, aplicada a un vecindario de la ciudad de Valdivia. El concepto se atribuye a la presencia de diversos cambios empleados en los distritos industriales, producto de las modificaciones en las políticas y economías, pero que actúan de modo distinto en diferentes zonas. En el texto se establece como ciudad del palimpsesto a Lo Sur, que inicialmente corres- 
ponde a una localidad netamente fructífera, lo que genera una identidad característica en la población, que, en definitiva, determinará el desarrollo urbano de la zona.

Posteriormente, Adriano Rovira se encarga de realizar un estudio sobre la expansión urbana en la ciudad de Puerto Montt. La relevancia de esta zona corresponde a su particular proceso de ocupación de la periferia, que ha concentrado un alto número de industrias, actividades de transporte y almacenamiento e incluso la presencia de parcelas agrorresidenciales. Junto a ello, la ciudad de Puerto Montt ha comenzado un proceso de diversificación de sus funciones que contribuye a originar una relación más fuerte con localidades cercanas y las invita a incrementar su número de habitantes.

Continuando con la ciudad de Puerto Montt, Hugo Marcelo Zunino analiza las nuevas formas estructurantes del territorio, a saber, la producción, redes sociales y de poder, que han sido consecuencia del desarrollo económico producto de la expansión en la producción acuícola y salmonera.

Para finalizar la sección y la obra por completo, se presenta un estudio de la huella ecológica de la Región de Magallanes y la Antártica Chilena, realizado por Luis Inostroza Pino, que se encarga de indicar el impacto que el ser humano provoca a nivel ambiental. A pesar de ser esta la zona con menor cantidad de población del país, el resultado de la huella ecológica impacta, pues prácticamente duplica el resultado a nivel nacional. En este sentido es que se hace urgente un Ilamado de atención para la localidad y el resto del país, con el propósito de la toma de conciencia respecto a la responsabilidad ambiental que se posee, más aún en zonas de importancia nacional y ambiental como esta.

Es así como la obra contiene una serie de estudios que se sumergen en las distintas modalidades en que se presentan los procesos de metropolización de las ciudades chilenas. Más allá de acusar las diferentes formas de transformación de las urbes, se enseñan las inevitables consecuencias sufridas por estos fenómenos. Los estudios se presentan no solo con el propósito de anunciarlos, sino también con el ideal de advertirlos y generar la intención de enfrentarlos con las herramientas correctas, contrarrestando sus efectos negativos, pues el desarrollo humano que genera los procesos de metropolización no tiende a detenerse. 